\title{
Homocysteine in Cerebrovascular Disease: an Independent Risk Factor for Subcortical Vascular Encephalopathy
}

Thomas Bertsch', Orell Mielke², Sabine Höly', Wilma Zimmer ${ }^{1}$, Wendy Casarin ${ }^{1}$, Johannes Aufenanger ${ }^{3}$, Silke Walter ${ }^{2}$, Frank Muehlhauser ${ }^{2}$, Sandra Kuehl², Andreas Ragoschke ${ }^{2}$ and Klaus Fassbender ${ }^{2}$

${ }^{1}$ Department of Clinical Chemistry,

2 Department of Neurology,

Clinic Mannheim, University of Heidelberg, Heidelberg, Germany

${ }^{3}$ Department of Laboratory Medicine,

Clinic Ingolstadt, Teaching Hospital of the Ludwig

Maximilians University Munich, Munich, Germany

Hyperhomocysteinemia is a risk factor for obstructive large-vessel disease. Here, we studied plasma concentrations of homocysteine and vitamins in patients suffering from subcortical vascular encephalopathy (SVE), a cerebral small-vessel disease leading to dementia. These results were compared to the homocysteine and vitamin plasma concentrations from patients with cerebral large vessel disease and healthy control subjects.

Plasma concentrations of homocysteine, vascular risk factors and vitamin status $\left(B_{6}, B_{12}\right.$, folate) were determined in 82 patients with subcortical vascular encephalopathy, in 144 patients with cerebral large-vessel disease and in 102 control subjects. Patients with SVE, but not those with cerebral large-vessel disease, exhibited pathologically increased homocysteine concentrations in comparison with control subjects without cerebrovascular disease. Patients with SVE also showed lower vitamin $B_{6}$ values in comparison to subjects without cerebrovascular disease. Logistic regression analysis showed that homocysteine is associated with the highest risk for SVE (odds ratio $5.7 ; \mathrm{Cl}$ 2.5-12.9) in comparison to other vascular risk factors such as hypertension, age and smoking.

These observations indicate that hyperhomocysteinemia is a strong independent risk factor for SVE.

Key words: Homocysteine; Vitamin $\mathrm{B}_{6}$; Vitamin $\mathrm{B}_{12}$; Cerebrovascular disease; Subcortical vascular encephalopathy; Dementia.

Abbrevations: $\mathrm{Cl}$, confidence interval; $\mathrm{CT}$, computed tomography; MRT, magnetic resonance tomography; SVE, subcortical vascular encephalopathy.

Introduction

Dementia caused by cerebrovascular disease is the second most frequent cause of dementia in older people after Alzheimer's disease. A subtype of vascular dementia, subcortical vascular encephalopathy (SVE) is character- ized by progressive memory deficits and cognitive decline, typical gait disorders, and incontinence (1). Morphological correlates for these neurological deficits are sclerosis and hyalinosis of small cerebral arteries and arterioles which are associated with diffuse periventricular white matter abnormalities and central lacunes demonstrable in computed or magnetic resonance to-
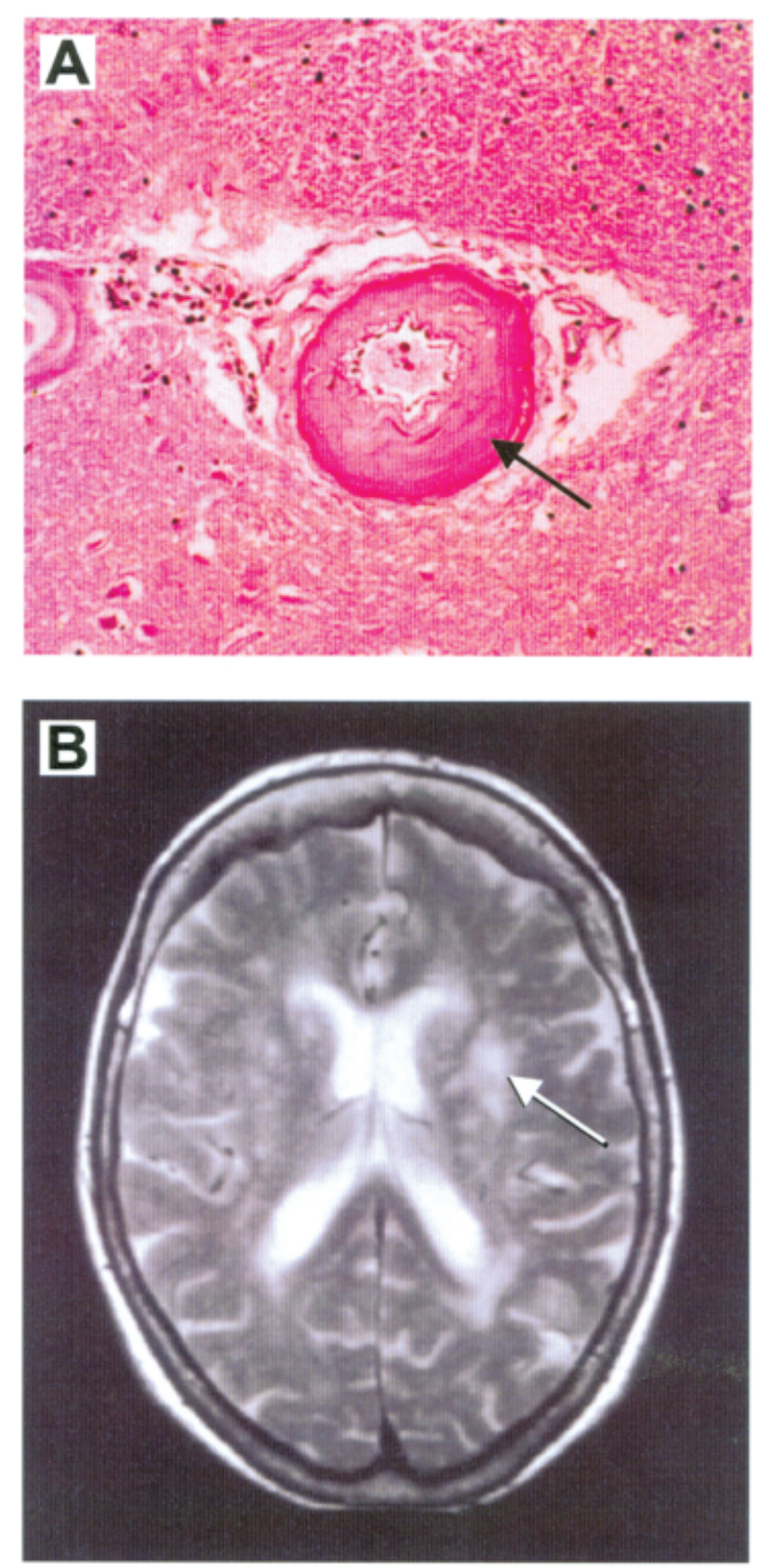

Fig. 1 A. Typical cerebral arterial vessel with hyalinosis and thickening of the media (arrow) from a patient who suffered from subcortical vascular encephalopathy (cerebral microangopathy). B. MRT-image from a patient with subcortical vascular encephalopathy showing white matter lesions (arrow). 
mography (Figure 1) (1, 2). Numerous studies have demonstrated elevated homocysteine concentrations in plasma as a risk factor for atherosclerosis of the coronary $(3,4)$, peripheral $(5)$ and cerebral blood vessels (6-9). Until now, no study has compared homocysteine plasma concentrations in cerebral macroangiopathy (large-vessel disease) and cerebral microangiopathy (small-vessel disease) associated with SVE. Therefore the aim of the present study was to investigate whether patients with distinct forms of cerebrovascular disease differ in their homocysteine concentrations in plasma.

\section{Patients and Methods}

\section{Patients and control subjects}

In all patients and healthy control subjects, history, clinical examination, extracranial and transcranial doppler, extracranial colour Doppler flow imaging and computed tomography (CT) or magnetic resonance imaging (MRI) were used to classify cerebrovascular disease. In 82 patients (37 females, 45 males; median age 73 years) from our clinic, SVE was diagnosed by combined information from standardized neurological, neuropsychological tests (including the Mini-Mental-State Examination (MMSE)) in all patients, and further assessments such as the Structured Interview for the Diagnosis of Dementia (SIDAM), Brief Assessment Interview (BAI) or Nuremberg Aging Inventory (NAI) in the majority of participants). Neuroradiological (CT or MRT) examination was performed according to the ICD-10 criteria (10). Symptoms such as typical gait disorders, urinary incontinence, or emotional disturbances were used to strengthen the diagnosis. This group was further divided into patients who suffered only from SVE $(n=51)$ and patients with combined SVE and large-vessel disease $(n=31)$. Additionally, 144 patients (55 females, 89 males; median age 67 years) with cerebral large-vessel disease (plaques or stenoses of the extracranial arteries and increase of cerebral blood flow velocity to $\geq 140 \mathrm{~cm} / \mathrm{s}$ in the large intracranial vessels or both) were studied. Controls were 102 people ( 54 females, 48 males; median age 65 years) without any cerebrovascular disease.

\section{Laboratory analysis}

Homocysteine and vitamin $B_{6}$ were measured in EDTAplasma by high-performance liquid chromatography (HPLC) with a Merck-Hitachi HPLC system from E. Merck, Darmstadt, Germany. Reagent kits were purchased from Medchrom, Heidelberg and Recipe, Munich, Germany. Folate and vitamin $B_{12}$ were measured with a microparticle-enzyme immunoassay on the IMX analyzer from Abbott, Wiesbaden, Germany.

\section{Statistics}

Spearman's correlation, Mann-Whitney U-test with a Bonferroni correction, and logistic regression analysis were used as statistic calculations.

\section{Results}

\section{Homocysteine and vitamins}

Significantly higher homocysteine concentrations were found in patients with SVE compared to controls and to patients with cerebral macroangiopathy only (Figure 2). Homocysteine concentrations were high, irrespective of concomitant cerebral macroangiopathy. Within the group of patients with SVE, homocysteine concentrations did not significantly differ between subjects with and without concomitant cerebral macroangiopathy (Figure 2). Interestingly, patients with only cerebral macroangiopathy did not exhibit significantly different homocysteine concentrations compared to control subjects without cerebrovascular disease. Patients with SVE showed significantly decreased plasma concentrations of vitamin $B_{6}$, but not of folate and $B_{12}$, in comparison with patients without cerebrovascular disease (Table 1).

\section{Risk factors}

Concerning the risk factor profile in our study population, hypertension was significantly more frequent in patients with cerebral microangipathy and in patients with macroangiopathy, compared to control subjects without cerebrovascular disease (Figure 3). Smoking was significantly more frequent in patients with cerebral macroangiopathy, but not in those with cerebral microangiopathy only, compared to subjects without cerebrovascular disease (Figure 3). The frequency of diabetes was equally distributed in each risk factor group (Figure 3).

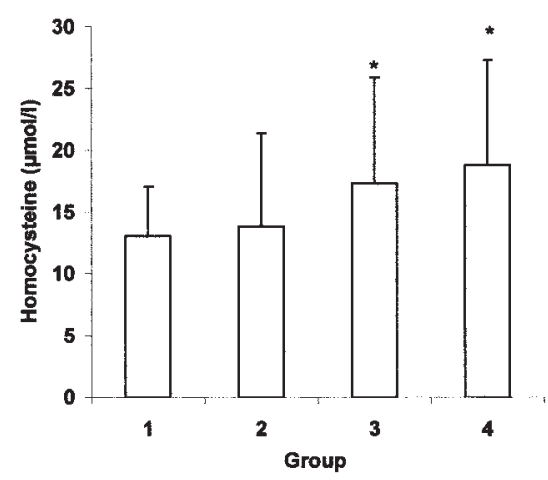

Fig. 2 Plasma levels of homocysteine (mean + SD) in the studied patients. Group 1, no cerebrovascular disease ( $n=102)$; Group 2, cerebral macroangiopathy ( $n=144)$; Group 3 , cerebral micro-and macroangiopathy ( $n=31$ ); Group 4 cerebral microangiopathy $(n=51)$. * Significant after Bonferroni correction compared with people without cerebrovascular disease. $\mathrm{p}<0.05$.

Tab. 1 Plasma concentrations of vitamins in the studies patients.

\begin{tabular}{lrlll}
\hline Group & $\mathrm{n}$ & $\mathrm{B} 6(\mathrm{nmol} / \mathrm{l})$ & $\mathrm{B}_{12}(\mathrm{pmol} / \mathrm{l})$ & Folate $(\mathrm{nmol} / \mathrm{l})$ \\
\hline 1 & 102 & $62.2 \pm 57.6$ & $333 \pm 199$ & $14.6 \pm 6.35$ \\
2 & 144 & $61.3 \pm 65.8$ & $269 \pm 109$ & $13.0 \pm 5.90$ \\
3 & 31 & $35.9 \pm 16.7^{*}$ & $272 \pm 181$ & $13.2 \pm 6.71$ \\
4 & 51 & $43.3 \pm 22.9^{*}$ & $275 \pm 160$ & $14.7 \pm 7.83$ \\
\hline
\end{tabular}

Group 1, no cerebrovascular disease; Group 2, cerebral macroangiopathy; Group 3, cerebral micro-and macroangiopathy; Group 4, cerebral microangiopathy. Values are give as mean \pm SD. * Significant after Bonferroni correction compared with people without cerebrovascular disease. $p<0.05$. 

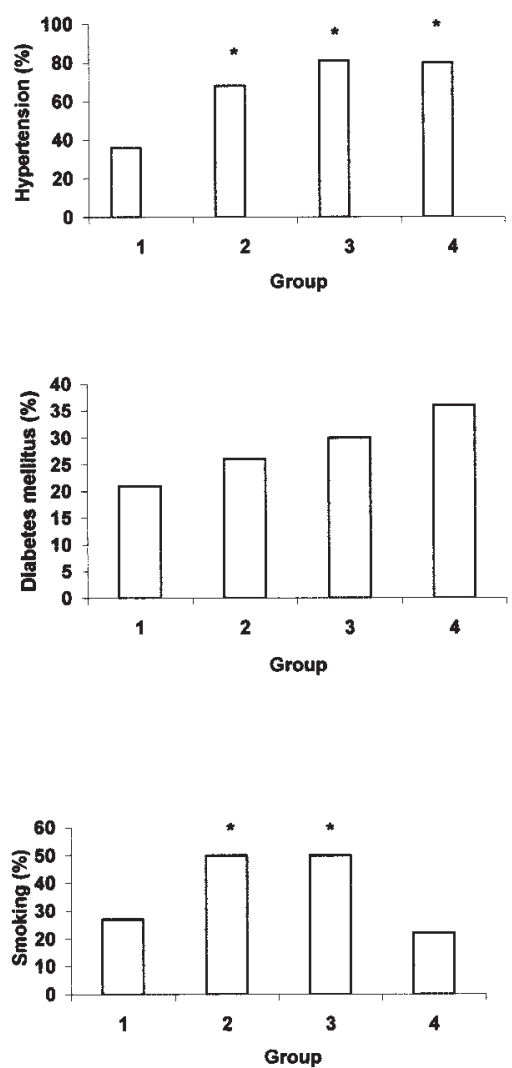

Fig. 3 Risk factor distribution in the studied patients. Group 1, no cerebrovascular disease $(n=102)$; Group 2, cerebral macroangiopathy ( $n=144)$; Group 3, cerebral micro-and macroangiopathy ( $n=31$ ); Group 4, cerebral microangiopathy $(n=51) . *$ Significant after Bonferroni correction compared with people without cerebrovascular disease. $p<0.05$.

Using logistic regression analysis with hyperhomocysteinemia, hypertension, age, smoking, and diabetes as variables, it could be demonstrated that hyperhomocysteinemia ( $\geq 15 \mu \mathrm{mol} / \mathrm{l}$ as used in earlier studies) is associated with the highest risk for SVE (odds ratio 5.7, $\mathrm{p}<0.0001,95 \% \mathrm{Cl} 2.5-12.9$ ) followed by hypertension $(5.2, \mathrm{p}<0.0001,95 \% \mathrm{Cl} 2.2-12.9)$, age (1.1, $\mathrm{p}<0.001,95 \% \mathrm{Cl} 1.0-1.1)$ and smoking (3.4, $\mathrm{p}<0.05,95 \% \mathrm{Cl} 1.3-9.2)$.

\section{Discussion}

Our study demonstrated that homocysteine concentration in the peripheral circulation is increased in patients with cerebral small-vessel disease, but not in those who suffer only from large-vessel disease as focused on in previous studies. This suggests that homocysteine injures the small penetrating cerebral arteries and arterioles rather than larger brain-supplying arteries. As thickening and hyalinosis of the media are important features of cerebral small vessel disease, homocysteine may be involved in the pathological activation of vascular smooth muscle and endothelial cells as has been shown in vitro (11-15).

Interestingly, logistic regression analysis showed that hyperhomocysteinemia is an independent risk factor for SVE that is even stronger than other vascular risk factors for SVE, e.g. hypertension and diabetes mellitus. Our findings are in accordance with previous reports on hyperhomocysteinemia in geriatric patients with psychiatric disorders (16, for review see 17), some of whom could have suffered from undiagnosed SVE, and in patients with diabetes mellitus (18) with microangiopathic complications. The association between smoking and SVE can be explained by the fact that smoking was frequently found in the subgroup of SVE patients with concomitant large-vessel disease. The frequency of smokers was not increased in patients who suffered from SVE without concomitant large vessel disease. This argues against a role for smoking as an important risk factor for SVE, but for a relationship between smoking and large-vessel disease which has been shown in numerous studies. As vitamins are involved in key positions in homocysteine metabolism (19) and as we found significantly lower homocysteine concentrations in our study population, it might be suggested that hypovitaminosis could have contributed to the development of SVE. Interestingly, we found that especially vitamin $B_{6}$ plasma levels were significantly lower in the group of patients suffering from SVE or SVE in combination with large-vessel disease. The role of vitamin $B_{6}$ in hyperhomocysteinemia is still under discussion but recently published studies showed that vitamin $B_{6}$ may have an influence on homocysteine plasma levels especially in combination with folate $(20,21)$. Also, animal studies indicate that vitamin $B_{6}$ deficiency is associated with higher homocysteine plasma concentrations and vascular disease (22). This possible important role of vitamin $B_{6}$ in homocysteine metabolism is at least in part supported by the results of our study.

Because cerebral microangiopathy and macroangiopathy can occur together, inclusion of patients with undiagnosed small-vessel disease in populations with carotid artery disease (large-vessel disease) might have resulted in artificially raised mean homocysteine concentrations in patients with large-vessel disease in earlier studies (6). This underscores the need to classify stroke syndromes and to identify patients with cerebral microangiopathy by exact neurological, neuropsychological, and brain imaging studies in future studies on hyperhomocysteinemia and cerebrovascular disease.

\section{Acknowledgements}

The histological picture of the cerebral arterial vessel was kindly provided by Dr. Clemens Sommer, Department of Neuropathology, Clinic Heidelberg, University of Heidelberg.

\section{References}

1. Ghika J, Bogousslavsky J. Subcortical arteriosclerotic encephalopathy (Binswanger's disease) In: Ginsberg MD, Bogosslavsky J, editors. Cerebrovascular disease -pathophys- 
iology, diagnosis and management. Malden Mass.: Blackwell 1998:1755-71.

2. Caplan LR. Binswanger's disease-revisited. Neurology $1995 ; 45: 626-33$.

3. Nygard O, Norderhaug JE, Refsum H, Ueland PM, Farstad $\mathrm{M}$, Vollset SE. Plasma homocysteine levels and mortality in patients with coronary artery disease. $\mathrm{N}$ Engl $\mathrm{J}$ Med $1997 ; 337: 230-6$.

4. Refsum H, Ueland PM, Nygard O, Vollset SE. Homocysteine and cardiovascular disease. Annu Rev Med 1998; 49:31-62.

5. Malinow MR, Kang SS, Taylor M, Wong PWK, Coull B, Inahara $\mathrm{T}$, et al. Prevalence of hyperhomocyst(e)inemia in patients with peripheral arterial occlusive disease. Circulation 1989; 79:1180-8.

6. Selhub J, Jaques PF, Bostom AG, D’ Agostino RB, Wilson $\mathrm{PW}$, Belanger AJ, et al. Association between plasma homocysteine concentrations and extracranial carotid-artery disease. N Engl J Med 1995; 332:286-91.

7. Perry IJ, Refsum H, Morris RW, Ebrahim SB, Ueland PM, Shaper AG. Prospective study of serum total homocysteine concentration and risk of stroke in middle-aged British men. Lancet 1995; 346:1395-8.

8. Spence JD, Malinow MR, Barnett PA, Marian AJ, Freeman $D$, Hegele RA. Plasma homocysteine concentration, but not MTHFR genotype, is associated with variation in carotid plaque area. Stroke 1999; 30:969-73.

9. Yoo JH, Chung CS, Kang Soo-Sang. Relation of plasma homocyst(e)ine to cerebral infarction and cerebral atherosclerosis. Stroke 1998; 29:2478-83.

10. WHO. International Classification of diseases and related health problems, tenth revision. Geneva, Switzerland: WHO 1993.

11. Van Guldener C, Stehouver CD. Hyperhomocysteinemia, vascular pathology, and endothelial dysfunction. Semin Thromb Hemost 2000; 26:281-9.

12. Mujumdar VS, Hayden MR, Tyagi SC. Homocyst(e)ine induces calcium second messenger in vascular smooth muscle cells. J Cell Physiol 2000; 183:28-36.

13. Chen C, Halkos ME, Surowiec SM, Conklin BS, Lin PH Lumsden AB. Effects of homocysteine on smooth muscle cell proliferation in both cell culture and artery perfusion culture model. J Surg Res 2000; 88:26-33.

14. Woo DK, Dudrick SJ, Sumpio BE. Homocysteine stimulates MAP kinase in bovine aortic smooth muscle cells. Surgery 2000; 128:59-66.
15. Wang G, Siow YL. Homocysteine stimulates nuclear factor kappaB activity and monocyte chemoattractant protein-1 expression in vascular smooth-muscle cells: a possible role for protein kinase C. Biochem J 2000; 352:817-26.

16. Nilsson K, Gustafson L, Faldt R, Andersson A, Brattstrom L, Lindgren $A$, et al. Hyperhomocysteinaemia - a common finding in a psychogeriatric population. Eur J Clin Invest 1996; 26:853-859.

17. Selhub J, Bagley LC, Miller J, Rosenberg IH. B vitamins, homocysteine, and neurocognitive function in the elderly. Am J Clin Nutr 2000; 71:Suppl:614-20.

18. Vaccaro O, Ingrosso D, Rivellese A, Greco G, Riccardi G. Moderate hyperhomocysteinemia and retinopathy in insulin-dependent diabetes. Lancet 1997; 349:1102-3.

19. Selhub J, Miller JW. The pathogenesis of homocysteinemia: interruption of coordinate regulation by S-adenosylmethionine of the remethylation and transsulfuration of homocysteine. Am J Clin Nutr 1992; 55:131-8.

20. McKinley MC, McNulty H, McPartlin J, Strain JJ, Petieva K, Ward M, et al. Low-dose vitamin B-6 effectively lowers fasting plasma homocysteine in healthy elderly persons who are folate and riboflavin replete. Am J Clin Nutr 2001; 73:759-64.

21. Mansoor MA, Kristensen O, Hervig T, Bates CJ, Pentieva K, Vefring $\mathrm{H}$, et al. Plasma total homocysteine response to oral doses of folic acid and pyridoxine hydrochloride (vitamin B6) in healthy individuals. Oral doses of vitamin B6 reduce concentrations of serum folate. Scand J Clin Lab Invest 1999; 59:139-46.

22. Smolin LA, Crenshaw TD, Kurtycz D, Benevenga. Homocyst(e)ine accumulation in pigs fed diets deficient in vitamin B6: relationship to atherosclerosis. J Nutr 1983; 113: 2022-33.

Received 19 April 2001, revised 17 July 2001, accepted 19 July 2001

Corresponding author: Dr. Thomas Bertsch, Klinikum Mannheim der Universität Heidelberg, Institut für Klinische Chemie, Theodor-Kutzer-Ufer 1-3, 68167 Mannheim, Germany

Fax: +49621383 3819,

E-mail: thomas.bertsch@ikc.ma.uni-heidelberg.de 